\title{
Concept Learning in Neuromorphic Vision Systems: What Can We Learn from Insects?
}

\author{
Fredrik Sandin', Asad I. Khan², Adrian G. Dyer,4, Anang Hudaya M. Amin', \\ Giacomo Indiveri ${ }^{6}$, Elisabetta Chicca ${ }^{7}$, Evgeny 0sipov ${ }^{8}$ \\ ${ }^{1}$ EISLAB, Luleå University of Technology, Luleå, Sweden \\ ${ }^{2}$ Clayton School of Information Technology, Monash University, Clayton, Australia \\ ${ }^{3}$ Department of Physiology, Monash University, Clayton, Australia \\ ${ }^{4}$ School of Media and Communication, Royal Melbourne Institute of Technology, Melbourne, Australia \\ ${ }^{5}$ Faculty of Information Science \& Technology (FIST), Multimedia University, Melaka, Malaysia \\ ${ }^{6}$ Institute of Neuroinformatics, University of Zurich and ETH Zurich, Zurich, Switzerland \\ ${ }^{7}$ Cognitive Interaction Technology, Center of Excellence, Bielefeld University, Bielefeld, Germany \\ ${ }^{8}$ Division of Computer Science, Luleå University of Technology, Luleå, Sweden \\ Email: fredrik.sandin@Itu.se, asad.khan@monash.edu, adrian.dyer@monash.edu, anang.amin@mmu.edu.my, \\ giacomo@ini.phys.ethz.ch, chicca@cit-ec.uni-bielefeld.de, evgeny.osipov@ltu.se
}

Received 25 March 2014; revised 20 April 2014; accepted 27 April 2014

Copyright (C) 2014 by authors and Scientific Research Publishing Inc.

This work is licensed under the Creative Commons Attribution International License (CC BY).

http://creativecommons.org/licenses/by/4.0/

c) (i) Open Access

\begin{abstract}
Vision systems that enable collision avoidance, localization and navigation in complex and uncertain environments are common in biology, but are extremely challenging to mimic in artificial electronic systems, in particular when size and power limitations apply. The development of neuromorphic electronic systems implementing models of biological sensory-motor systems in silicon is one promising approach to addressing these challenges. Concept learning is a central part of animal cognition that enables appropriate motor response in novel situations by generalization of former experience, possibly from a few examples. These aspects make concept learning a challenging and important problem. Learning methods in computer vision are typically inspired by mammals, but recent studies of insects motivate an interesting complementary research direction. There are several remarkable results showing that honeybees can learn to master abstract concepts, providing a road map for future work to allow direct comparisons between bio-inspired computing architectures and information processing in miniaturized "real" brains. Considering that the brain of a bee has less than $\mathbf{0 . 0 1 \%}$ as many neurons as a human brain, the task to infer a minimal architecture and mechanism of concept learning from studies of bees appears well motivated. The relatively low complexity of insect sensory-motor systems makes them an interesting model for the further development of bio-inspired computing architectures, in particular for resource-constrained applications such as miniature robots, wireless sensors and handheld or wearable devices. Work in that direction is a natural step towards understanding and making use
\end{abstract}


of prototype circuits for concept learning, which eventually may also help us to understand the more complex learning circuits of the human brain. By adapting concept learning mechanisms to a polymorphic computing framework we could possibly create large-scale decentralized computer vision systems, for example in the form of wireless sensor networks.

\section{Keywords}

\section{Concept Learning, Computer Vision, Computer Architecture, Neuromorphic Engineering, Insect}

\section{Introduction}

Efficient and lightweight computer vision devices that operate on a low energy budget can open up novel application domains. For instance, reliable collision avoidance, localization, and navigation have a range of practical applications. Making systems that can perform such tasks in environments with greater complexity and uncertainty than current systems can tolerate is an important challenge [1].

Neuromorphic [2] vision sensors are hybrid analog/digital VLSI devices that implement models of biological visual systems in hardware [3]-[7]. Similarly, neuromorphic neural processing systems implement real-time biologically plausible models of neural circuits and architectures that can be configured to carry out complex signal processing and computational tasks. Systems composed of neuromorphic vision sensors and neuromorphic multi-neuron chips are becoming elaborate and powerful enough for use in real-world applications [8] [9]. For example, a system of this type has been recently synthesized to perform context-dependent classification of motion patterns observed by a silicon retina [10]. Neuromorphic systems are designed to deal with uncertainties and show potential for brain-like computability and remarkable capabilities in vision, for example in terms of high temporal resolution and dynamic range at low data rate and power use. The speed at which such a system can operate in resource-constrained environments surpasses that of conventional computer vision systems. A major challenge, however, is to design a flexible sensory-motor architecture that can be adapted to real-world applications in a cost-efficient manner.

Seeking inspiration in animal cognition, it is evident that concept learning [11] [12] is a key function that enables motor response in complex environments and novel situations by generalization of former experience, thereby making it unnecessary to learn each particular situation that is encountered. There is evidence for three types of concept learning in animals [12]: similarity-based in which items are categorized based on similarity; relational in which one item is categorized relative to another; associative in which arbitrary stimuli become interchangeable due to association with another stimulus or response. Concept learning is also a central part of analogy making [11] [13], which is used to infer novel information about objects or situations through mapping of memories with similar compositional structure, see [14] [15] for examples. Learning approaches to computer vision are typically inspired by empirical studies of vision in mammals. A complementary approach is to seek inspiration and guidance in studies of insects that demonstrate conceptual learning and problem solving abilities [16]-[18]. Work in that direction is motivated due to the relatively low complexity of insect brains, e.g., $10^{5}$ neurons in the fruit fly brain [19] or $10^{6}$ in the bee brain [20] versus $10^{11}$ in the human brain [21]. Despite the low number of neurons, the mushroom bodies in the brain of honeybees process visual, olfactory, gustatory, and mechanosensory information, and show a remarkable ability to learn how to solve problems [22]. Insect brains provide an excellent template for an empirical study, and for electronic implementation using neuromorphic circuits. For example, some aspects of the silicon retina vision sensors correlate to insect vision-prioritizing speed of processing over finer details such as colour vision.

Being inspired by neural systems, the neuromorphic engineering approach is naturally motivated for the development of compact low-power sensory-motor and neural processing systems. In distributed and heterogeneous large-scale systems the polymorphic computing $(\mathrm{PmC})$ approach provides a scalable and reliable alternative to conventional computing architectures for pattern recognition, and vector symbolic architectures (VSAs) provide a mathematical framework for encoding concepts and related functions with distributed representations, which in principle can be implemented in neuromorphic devices and systems. In the next section we introduce the remarkable concept learning results obtained in studies of bees that are mentioned above. Thereafter we 
briefly introduce the neuromorphic approach to building artificial sensory-motor systems, followed by an introduction to vector symbolic representations of concepts and polymorphic computing in decentralized systems.

\section{Higher-Order Learning in Insects}

Insects operate in complex environments and potentially provide a valuable model for understanding how information is efficiently managed in a miniaturized brain [23] [24]. In particular the honeybee has become a supermodel [18] [22] [25]-[27] for understanding learning, due to their altruistic lifestyle that provides convenient access to investigating how individuals learn through experience to solve complex problems. Such learning was first demonstrated by the Nobel laureate Karl von Frisch exactly 100 years ago [28] who showed that bees could be trained to solve a visual task through associative conditioning with a rewarding sweet solution, and tested in extinction trials when rewards were removed. Since bees collect nutrition to contribute to the entire colony, it is possible to train and test one individual bee for 8 - 10 hours a day to evaluate learning in psychophysics type experiments, where behavioral outcomes to known stimuli allow for black box evaluations of information processing by the visual system [29].

The brain of the bee has been intensively studied and is known to have very distinct hierarchical structures [30] [31]. Considering visual stimuli, the honeybee as a model of bee vision has three classes of receptors (UV-, Blue- and Green-sensitive). The Green receptor signals are initially processed in the lamina region of the brain, which has neurons with fast response times that potentially drive rapid responses to achromatic signals like salient edges [32]. Beyond the level of the lamina, visual processing incorporates all three classes of receptor inputs that occur in specialized regions of the honeybee brain which have been imaged at high resolution [30]. After the lamina, visual signals are next processed in the medulla where information starts to become segregated by specialized neurons including broad-band neurons that respond equally to multiple wavelengths of light; narrow-band neurons that respond to input from a single photoreceptor type; or colour-opponent-neurons that antagonistically process multiple spectrally different signals [31]. Interestingly, it appears that from this medulla level of processing, signals may follow different pathways such that neurons from the outer layers of the medulla project to the posterior protocerebrum region of the brain, whilst signals processed in the inner layers of the medulla project to the lobula, the lateral protocerebrum and mushroom bodies. These different pathways may enable either fast hard-wired responses, or a capacity to learn through experience [31]. Thus whilst the bee brain contains less than one million neurons, the hierarchical structure and alternative neural pathways appear to facilitate either a hard wired or plastic learning capacity for different scenarios that might occur for a flying insect.

Indeed individual honeybees show a remarkable capacity to learn how to solve problems. One of the clearest demonstrations of this is the capacity of honeybees to solve delayed matching-to-sample type tasks where a viewed initial stimulus must be loaded into working memory, and then subsequently compared to alternatives in order to make a correct decision and collect a sucrose solution reward [33]. Whilst this type of delayed matching to sample task actually takes an individual bee a very long time to learn with variable stimuli during different trials; once the "matching" rule is learnt (involving long term memory), a bee can quickly apply the acquired rule to a novel task like matching scents within a trail [33]. Indeed, rule learning appears to be a major way that insects learn to solve problems, but only if provided with the correct conditions to reinforce flexible learning. For example, if free flying honeybees have to "only" learn a fixed target at a constant visual angle, then there is a poor ability to make correct decision if conditions change, but bees trained to a set of variable stimuli learn how to use this acquired information to solve novel problems [34]. Bees can learn to solve complex visual tasks like face recognition using configural processing mechanisms [35], and even with the right conditioning experience deal with complex transformation imposed by viewpoint variation [36]. Some of these tasks like solving above/below relational problems [16], or simultaneously applying multiple rules like spatial relationships and differences [17], are at a level of complexity that challenges our current understanding of what mammalian brains can achieve [18] [37]. This shows that miniaturized information-processing systems have a capacity to efficiently deal with very complex information provided that they are appropriately organized through adaptation. However, current challenges exist in completely bridging between the higher-order learning (i.e. non-elemental learning) behavior demonstrated in free-flying bees, and our complete understanding of the neural regions responsible because when a bee is harnessed, as would typically be required to enable brain recordings [38], learning performance for visual stimuli in significantly impaired [39]. Solutions to this problem are starting 
to emerge with new work on displaying complex moving stimuli to tethered bees [40], or using closed-loop paradigms allowing tethered but walking bees to actively control visual objects within a virtual reality arena [41]. Indeed such experiments reveal that attention like behavioral responses result in modulation of neural activity in the medulla region of the brain, again pointing towards the importance of this structure for filtering information for decision making in insects. These remarkable studies provide a road map for future work to more fully allow direct comparisons between bio-inspired computing architectures and information processing in miniaturized "real" brains [24] [31]. Currently there appears to be similarities in hierarchical organization and segregation of information parsed into fast hard-wired solutions, or more plastic learning modules depending upon task difficulty.

\section{Neuromorphic Sensory-Motor Systems}

Neuromorphic vision sensors are hybrid analog/digital VLSI devices that implement hardware models of biological visual systems, which typically are used in machine vision [3]-[7]. It is only recently that these hardware models have become elaborate enough for use in a variety of engineering applications [8] [9]. These types of devices and systems offer a low cost alternative to special purpose Digital Signal Processors (DSPs) for machine vision tasks. They can be used for reducing the computational load on the digital system in which they are embedded or, ideally, for carrying out all of the necessary computation without the need of any additional hardware. They process images directly at the focal plane level: each pixel contains local circuitry that performs different types of spatio-temporal computations on the continuous analog brightness signal in real-time. In contrast, CCD cameras or conventional CMOS imagers merely measure the brightness at the pixel level, eventually adjusting their gain to the average brightness level of the whole scene. In neuromorphic vision chips, photo-receptors, memory elements and computational nodes share the same physical space on the silicon surface. The specific computational function of a neuromorphic sensor is determined by the structure of its architecture and by the way its pixels are interconnected. Since each pixel processes information based on locally sensed signals and on data arriving from its neighbors, the type of computation being performed is fully parallel and distributed. Another important feature is the asynchronous operation of neuromorphic sensors, which is preferable to clocked operation for sensory processing, given the continuous nature of sensory signals. Clocked systems introduce temporal aliasing artifacts that can significantly compromise the time-dependent computations performed in real-time sensory processing systems.

Recent neuromorphic vision sensors are clock-less and use a frame-less communication protocol [42]-[44]. In these sensors each pixel is assigned an address, and when a pixel generates an event (e.g., when it measures a contrast difference greater than a set threshold) its address is instantaneously put on a digital bus, using asynchronous logic. In this asynchronous “Address-Event Representation” (AER) time represents itself, and analog signals are encoded by the inter-spike intervals between the addresses of their sending nodes. Address-events are the digital pulses written on the bus. In this way neuromorphic multi-chip systems can be assembled, consisting for example of neuromorphic sensory devices such as silicon retinas interfaced to one or more chips containing networks of spiking neuron circuits. Spiking neural network chips can receive address-events produced by neuromorphic sensors and process them (e.g., to implement concept learning), and eventually transmit the processed signals to actuators, thus implementing complete neuromorphic sensory-motor systems.

\section{Representation of Concepts and Conceptual Relationships}

In general, the question how to realize higher-order learning and cognition in neuromorphic sensory-motor systems is open because we do not fully understand the principles and architectures of neural circuits in brains that make this possible. In particular, extracting and making use of conceptual relationships such as same/different or above/below is a challenge. Computational approaches to analyze spatial structure of images typically result in NP-hard graph matching problems [45] that are difficult to approximate. Research over the last few decades have advanced our understanding of concept learning [11]-[13] [46], but we are still lacking a plausible description of the mechanisms and neural architecture involved. Open questions include the nature of neural object representations and how representations change across different processing stages, for example how object features are computationally integrated into coherent object representations, and how these are read-out by higherorder circuits. This problem includes learning of elementary sparse representations of objects and events, and representations of invariant features of object and event categories, which are grounded in sensory projections 
[47] [48]. Generative representations and motor programs play an important role in concept learning, see for example [49] and references therein. This appears natural in the case of animals since concept learning is an integrated part of the sensory-motor system, but covert actions may be more important than overt actions and the underlying computational mechanisms are unknown. In principle, the possibilities to generalize beyond familiar examples suggest that concepts are representationally rich. Therefore, it is remarkable that relatively few examples can be sufficient to learn a new concept. Efficient sensory and motor-program coding strategies are likely involved, for example sparse coding [50] and complexity minimization [51].

Vector symbolic architectures (VSAs) [52]-[54] offer an interesting mathematical framework for modeling of concepts, relationships between concepts and analogies [14] [15]. VSAs are based on high-dimensional vector representations of objects (e.g., image parts), relations (e.g., composition of objects), and sequences (e.g., sentence structures), and operators for dynamic binding and formation of chunks of multiple concepts in working memory. Such representations can in principle be integrated in spiking neural networks and neuromorphic hardware, see [55] for an example where a large-scale brain model is designed and simulated. VSAs can be integrated with a model of associative memory [14] known as sparse distributed memory [56] (SDM), forming a Monte Carlo importance sampler that approximates Bayesian inference [57]. The integration of an associative memory enables learning of multiple concepts and relationships. VSAs offer possibilities to prove learnability and enable rapid learning and generalization with high systematicity, meaning that generalization to compositional structures more complex than those in the training set is possible [58]. However, it is not known to what extent a VSA can approximate the complex dynamics of neural representations read out by higher-order circuits of a brain. One fundamental aspect of VSAs and the SDM is that similar or related concepts are represented by similar vectors in a vector space, which appears to be coherent with electrophysiology of hippocampal place cells in rodents showing that the topology of the stimulus space can be inferred from co-firing neurons [59].

Research integrating computational and empirical approaches to study the neural mechanisms underlying object perception and concept learning as it is observed in the behaviour of bees can help us to address the challenging questions outlined above, and to develop a more realistic architecture and computational model of concept learning. Insights gained from such studies can also stimulate the development of artificial concept learning mechanisms for decentralized applications such as wireless sensor networks (WSNs) and the Internet, for example in the form of polymorphic computing which is briefly introduced in the next section.

Extending VSA-based concept learning to the case of distributed networks opens a possibility to develop drastically new communication modes. For example, the superposition of broadcasted messages in a wireless transmission medium could be exploited as a physical implementation of the VSA chunking mechanism rather than as a collision, thereby dynamically creating new concepts in the form of parallel transmissions. Recently a VSA was adopted for implementing novel communication protocols and architectures for collective communications in machine-to-machine communication scenarios including wireless sensor networks [60] [61]. The first work demonstrates unique reliability and timing properties that are essential in the context of industrial machine-to-machine communications. The latter work presents an example of collective communications using current radio technology.

\section{Decentralized Computer Vision Systems}

In a decentralized computer vision system the transportation of data to a centralized location for information processing is inefficient in terms of energy use and communication, and it entails unnecessary time penalties. Ideally pattern recognition should commence as soon as sensory data enters the processing network. The ability to perform such computations, however, depends on the availability of a network-centric computational model that is fully distributable and thus able to dynamically reconfigure its internal resources. Changing the hardware architecture to suit computational goals is common in e.g. field programmable gate arrays (FPGAs), but is less explored in the context of networks and pattern learning in complex and changing environments.

Polymorphic computing (PmC) is a scalable and reliable alternative to conventional computing architectures for pattern recognition in such scenarios. Polymorphic computing can in this context be defined as a computing architecture that can vigorously adapt towards changes in the computational model requirements of specific applications. A key characteristic of a polymorphic computer is the ability to dynamically re-arrange the hardware configuration during runtime [62]. Ideally it should also be possible to dynamically change the software during runtime. A benefit of PmC is the ability to dynamically divide and distribute tasks according to time-varying computational capacities and requirements. The concept of making such a machine can be traced back to late 
1950s [63], but only a few practical design concepts and methods have emerged so far. One instance of a reconfigurable computer design is the MONARCH (Morphable Networked Micro-Architecture) system developed at the University of Southern California and Raytheon Inc. [64].

WSNs and the emerging Internet of Things offer new interesting opportunities to develop and study such systems, possibly taking a bio-inspired approach. For example, the dynamic reconfigurablity of PmC through optimal dataflow manipulation in a network mimics learning-induced plasticity in brains. A distributed pattern recognition approach developed for WSNs is the hierarchical graph neuron (HGN) [65], which implements one-shot learning of patterns and provisions the structure necessary for deep learning (a form of learning that models data at multiple levels of abstraction or composition). Deep learning, which apparently is nature's way of coping with complexity in visual processing, is based on hierarchically connected layers, with local feature learning at the lowest layer and upper layers combining features into higher-order representations. A similar hierarchical organization of information takes place in the visual system of honeybees. In principle, a suitably designed polymorphic computer with stimuli-driven data flows could facilitate deep learning, and by using VSA principles for the design of the higher-order architecture, such a system could be capable of processing concepts. The HGN has been further developed at the conceptual level to a reconfigurable PmC design [66]. In our approach pattern recognition is undertaken progressively in multiple layers as sensory data flows from the input layer(s) upwards in the hierarchy. This design envisages an efficient in-network pattern recognition approach. One way to readily demonstrate the concept will be accomplished by implementing a connection-based computing mechanism within a WSN. Doing so will facilitate computation through data flows between simple processing elements.

\section{Concluding Remarks}

Systems composed of neuromorphic vision sensors and neuromorphic multi-neuron chips are becoming elaborate enough for use in real-world applications [8]-[10]. If higher-order learning and cognitive mechanisms can be implemented in such systems, it will enable efficient and lightweight computer vision systems that can perform tasks in environments with greater complexity and uncertainty than current systems can tolerate. Concept learning [11] [12] is a central part of animal cognition that enables appropriate motor response in novel situations by generalization of former experience, possibly from a few training examples. These aspects make concept learning a challenging and important problem, which can lead to discoveries that radically change how we design and use computer vision systems and computing architectures. Learning methods in computer vision are typically inspired by mammals, but recent studies of insects motivate an interesting complementary research direction that is outlined in this paper. In particular, individual honeybees show a remarkable capacity to learn how to solve problems involving abstract concepts. Some of these tasks like solving above/below relational problems [16], or simultaneously applying multiple rules like spatial relationships and differences [17], are at a level of complexity that challenges our current understanding of what mammalian brains can achieve [18] [37]. These results provide a road map for future work to allow direct comparisons between bio-inspired computing architectures and information processing in miniaturized "real" brains. Considering that the brain of a bee has less than $0.01 \%$ as many neurons as a human brain, the task to infer a minimal neural architecture and mechanism mediating concept learning and other forms of higher-order learning from studies of bees appears well motivated. Vector symbolic architectures [52]-[54] offer a mathematical framework for modeling of concepts and higher-order learning with distributed representations, which can also be implemented in digital processing systems and neuromorphic systems. The integration of concept learning mechanisms in a polymorphic computing architecture could enable the development of decentralized computer vision systems, for example in the form of wireless sensor networks, which are scalable and can perform advanced pattern recognition in complex and changing environments.

\section{Acknowledgements}

This work is partially supported by the Swedish Foundation for International Cooperation in Research and Higher Education (STINT), grant number IG2011-2025. AK thank Ahmet Sekercioglu and Alexander Senior for their assistance in the preparation of this paper. AGD thanks ARC DP0878968/DP0987989 for funding support. 


\section{References}

[1] (2013) A Roadmap for U.S. Robotics: From Internet to Robotics. tech. rep. www.robotics-vo.us

[2] Indiveri, G. and Horiuchi, T.K. (2011) Frontiers in Neuromorphic Engineering. Frontiers in Neuroscience, 5, 118.

[3] Mahowald, M. and Mead, C. (1989) Analog VLSI and Neural Systems. Silicon Retina, Addison-Wesley, Reading, 257-278.

[4] Boahen, K. and Andreou, A. (1992) A Contrast Sensitive Silicon Retina with Reciprocal Synapses. In: Moody, J., Hanson, S. and Lippman, R., Eds., Advances in Neural Information Processing Systems (Vol. 4), Morgan Kaufmann, San Mateo, 764-772.

[5] Kramer, J. (2002) An Integrated Optical Transient Sensor. IEEE Transactions on Circuits and Systems II, 49, $612-628$. http://dx.doi.org/10.1109/TCSII.2002.807270

[6] Lichtsteiner, P., Posch, C. and Delbruck, T. (2008) An $128 \times 128120$ dB $15 \mu$ s Latency Temporal Contrast Vision Sensor. IEEE Journal of Solid State Circuits, 43, 566-576. http://dx.doi.org/10.1109/JSSC.2007.914337

[7] Posch, C., Matolin, D. and Wohlgenannt, R. (2011) A QVGA 143 dB Dynamic Range Frame-Free PWM Image Sensor with Lossless Pixel-Level Video Compression and Time-Domain CDS. IEEE Journal of Solid-State Circuits, 46, 259-275. http://dx.doi.org/10.1109/JSSC.2010.2085952

[8] Koch, C. and Mathur, B. (1996) Neuromorphic Vision Chips. IEEE Spectrum, 33, 38-46. http://dx.doi.org/10.1109/6.490055

[9] Liu, S.-C. and Delbruck, T. (2010) Neuromorphic Sensory Systems. Current Opinion in Neurobiology, 20, $288-295$. http://dx.doi.org/10.1016/j.conb.2010.03.007

[10] Neftci, E., Binas, J., Rutishauser, U., Chicca, E., Indiveri, G. and Douglas, R.J. (2013) Synthesizing Cognition in Neuromorphic Electronic Systems. Proceedings of the National Academy of Sciences, 110, E3468-E3476. http://dx.doi.org/10.1073/pnas.1212083110

[11] Zentall, T.R., Wasserman, E.A., Lazareva, O.F., Thompson, R.R.K. and Ratterman, M.J. (2008) Concept Learning in Animals. Comparative Cognition \& Behavior Reviews, 3, 13-45.

[12] Zentall, T.R., Wasserman, E.A. and Urcuioli, P.J. (2014) Associative Concept Learning in Animals. Journal of the Experimental Analysis of Behavior, 101, 130-151. http://dx.doi.org/10.1002/jeab.55

[13] Gentner, D. and Smith, L.A. (2013) Analogical Learning and Reasoning. In: The Oxford Handbook of Cognitive Psychology, Oxford University Press, Oxford, 668-681.

[14] Emruli, B. and Sandin, F. (2014) Analogical Mapping with Sparse Distributed Memory: A Simple Model That Learns to Generalize from Examples. Cognitive Computation, 6, 74-88.

[15] Emruli, B., Gayler, R. and Sandin, F. (2013) Analogical Mapping and Inference with Binary Spatter Codes and Sparse Distributed Memory. The 2013 International Joint Conference on Neural Networks (IJCNN), Dallas, 4-9 August 2013, 1-8

[16] Avarguès-Weber, A., Dyer, A.G. and Giurfa, M. (2011) Conceptualization of Above and Below Relationships by an Insect. Proceedings of the Royal Society B: Biological Sciences, 278, 898-905.

[17] Avarguès-Weber, A., Dyer, A.G., Combe, M. and Giurfa, M. (2012) Simultaneous Mastering of Two Abstract Concepts by the Miniature Brain of Bees. Proceedings of the National Academy of Sciences, 109, 7481-7486. http://dx.doi.org/10.1073/pnas.1202576109

[18] Avarguès-Weber, A. and Giurfa, M. (2013) Conceptual Learning by Miniature Brains. Proceedings of the Royal Society B: Biological Sciences, 280. http://dx.doi.org/10.1098/rspb.2013.1907

[19] Chiang, A.S., Lin, C.Y., Chuang, C.C., Chang, H.M., Hsieh, C.H., Yeh, C.W., Shih, C.T., Wu, J.J., Wang, G.T., Chen, Y.C., Wu, C.C., Chen, G.Y., Ching, Y.T., Lee, P.C., Lin, C.Y., Lin, H.H., Wu, C.C., Hsu, H.W., Huang, Y.A., Chen, J.Y., Chiang, H.J., Lu, C.F., Ni, R.F., Yeh, C.Y. and Hwang, J.K. (2011) Three-Dimensional Reconstruction of BrainWide Wiring Networks in Drosophila at Single-Cell Resolution. Current Biology, 21, 1-11. http://dx.doi.org/10.1016/j.cub.2010.11.056

[20] Witthöft, W. (1967) Absolute anzahl und verteilung der zellenim him der honigbiene. Zeitschrift für Morphologie der Tiere, 61, 160-184.

[21] Herculano-Houzel, S. (2009) The Human Brain in Numbers: A Linearly Scaled-Up Primate Brain. Frontiers in Human Neuroscience, 3, 31.

[22] Giurfa, M. (2013) Cognition with Few Neurons: Higher-Order Learning in Insects. Trends in Neurosciences, 36, 285294. http://dx.doi.org/10.1016/j.tins.2012.12.011

[23] Ibbotson, M. (2001) Evidence for Velocity-Tuned Motion-Sensitive Descending Neurons in the Honeybee. Proceedings of the Royal Society B: Biological Sciences, 268, 2195-2201. http://dx.doi.org/10.1098/rspb.2001.1770 
[24] Srinivasan, M.V. (2010) Honey Bees as a Model for Vision, Perception, and Cognition. Annual Review of Entomology, 55, 267-284. http://dx.doi.org/10.1146/annurev.ento.010908.164537

[25] Reinhard, J., Srinivasan, M.V. and Zhang, S.W. (2004) Olfaction: Scent-Triggered Navigation in Honeybees. Nature, 427, 411. http://dx.doi.org/10.1038/427411a

[26] Srinivasan, M.V., Zhang, S., Altwein, M. and Tautz, J. (2000) Honeybee Navigation: Nature and Calibration of the "Odometer”. Science, 287, 851-853. http://dx.doi.org/10.1126/science.287.5454.851

[27] Esch, H.E., Zhang, S.W., Srinivasan, M.V. and Tautz, J. (2001) Honeybee Dances Communicate Distances Measured by Optic Flow. Nature, 411, 581-583. http://dx.doi.org/10.1038/35079072

[28] von Frisch, K. (1914) Der Farbensinn und Formensinn der Biene. Fischer, Jena.

[29] Dyer, A.G., Spaethe, J. and Prack, S. (2008) Comparative Psychophysics of Bumblebee and Honeybee Colour Discrimination and Object Detection. Journal of Comparative Physiology A, 194, 617-627. http://dx.doi.org/10.1007/s00359-008-0335-1

[30] Brandt, R., Rohlfing, T., Rybak, J., Krofczik, S., Maye, A., Westerhoff, M., Hege, H.C. and Menzel, R. (2005) ThreeDimensional Average-Shape Atlas of the Honeybee Brain and Its Applications. The Journal of Comparative Neurology, 492, 1-19. http://dx.doi.org/10.1002/cne.20644

[31] Dyer, A.G., Paulk, A.C. and Reser, D.H. (2011) Colour Processing in Complex Environments: Insights from the Visual System of Bees. Proceedings of the Royal Society B: Biological Sciences, 278, 952-959.

[32] Skorupski, P. and Chittka, L. (2010) Differences in Photoreceptor Processing Speed for Chromatic and Achromatic Vision in the Bumblebee, Bombust errestris. The Journal of Neuroscience, 30, 3896-3903. http://dx.doi.org/10.1523/JNEUROSCI.5700-09.2010

[33] Giurfa, M., Zhang, S., Jenett, A., Menzel, R. and Srinivasan, M.V. (2001) The Concepts of "Sameness” and "Difference” in an Insect. Nature, 410, 930-933. http://dx.doi.org/10.1038/35073582

[34] Dyer, A.G. and Griffiths, D.W. (2012) Seeing Near and Seeing Far; Behavioural Evidence for Dual Mechanisms of Pattern Vision in the Honeybee (Apis mellifera). The Journal of Experimental Biology, 215, 397-404. http://dx.doi.org/10.1242/jeb.060954

[35] Avarguès-Weber, A., Portelli, G., Benard, J., Dyer, A. and Giurfa, M. (2010) Configural Processing Enables Discrimination and Categorization of Face-Like Stimuli in Honeybees. The Journal of Experimental Biology, 213, 593-601. http://dx.doi.org/10.1242/jeb.039263

[36] Dyer, A.G. and Vuong, Q.C. (2008) Insect Brains Use Image Interpolation Mechanisms to Recognize Rotated Objects. PLOS ONE, 3, e4086. http://dx.doi.org/10.1371/journal.pone.0004086

[37] Chittka, L. and Niven, J. (2009) Are Bigger Brains Better? Current Biology, 19, R995-R1008. http://dx.doi.org/10.1016/j.cub.2009.08.023

[38] Paulk, A.C., Dacks, A.M., Phillips-Portillo, J., Fellous, J.M. and Gronenberg, W. (2009) Visual Processing in the Central Bee Brain. The Journal of Neuroscience, 29, 9987-9999. http://dx.doi.org/10.1523/JNEUROSCI.1325-09.2009

[39] Niggebrügge, C., Leboulle, G., Menzel, R., Komischke, B. and de Ibarra, N.H. (2009) Fast Learning but Coarse Discrimination of Colors in Restrained Honeybees. Journal of Experimental Biology, 212, 1344-1350. http://dx.doi.org/10.1242/jeb.021881

[40] Luu, T., Cheung, A., Ball, D. and Srinivasan, M.V. (2011) Honeybee Flight: A Novel “Streamlining” Response. The Journal of Experimental Biology, 214, 2215-2225. http://dx.doi.org/10.1242/jeb.050310

[41] Paulk, A.C., Stacey, J.A., Pearson, T.W.J., Taylor, G.J., Moore, R.J.D., Srinivasan, M.V. and van Swinderen, B. (2014) Selective Attention in the Honeybee Optic Lobes Precedes Behavioral Choices. Proceedings of the National Academy of Sciences of the United States of America, 111, 5006-5011. http://dx.doi.org/10.1073/pnas.1323297111

[42] Delbruck, T. (2008) Frame-Free Dynamic Digital Vision. Proceedings of International Symposium on Secure-Life Electronics Advanced Electronics for Quality Life and Society, Tokyo, 6-7 March 2008, 21-26.

[43] Posch, C., Matolin, D., Wohlgenannt, R., Hofstätter, M., Schön, P., Litzenberger, M., Bauer, D. and Garn, H. (2010) Biomimetic Frame-Free HDR Camera with Event-Driven PWM Image/Video Sensor and Full-Custom Address-Event Processor. 2010 IEEE Biomedical Circuits and Systems Conference (BioCAS), Paphos, 3-5 November 2010, 254-257.

[44] Benosman, R., Ieng, S.H., Clercq, C., Bartolozzi, C. and Srinivasan, M. (2012) Asynchronous Frameless Event-Based Optical Flow. Neural Networks, 27, 32-37. http://dx.doi.org/10.1016/j.neunet.2011.11.001

[45] Conte, D., Foggia, P., Sansone, C. and Vento, M. (2004) Thirty Years of Graph Matching in Pattern Recognition. International Journal of Pattern Recognition and Artificial Intelligence, 18, 265-298. http://dx.doi.org/10.1142/S0218001404003228

[46] Jia, Y., Abbott, J.T., Austerweil, J., Griffiths, T. and Darrell, T. (2013) Visual Concept Learning: Combining Machine 
Vision and Bayesian Generalization on Concept Hierarchies. In: Burges, C., Bottou, L., Welling, M., Ghahramani, Z. and Weinberger, K., Eds., Advances in Neural Information Processing Systems, 26, 1842-1850.

[47] Harnad, S. (1990) The Symbol Grounding Problem. Physica D: Nonlinear Phenomena, 42, 335-346. http://dx.doi.org/10.1016/0167-2789(90)90087-6

[48] Barsalou, L.W. (2008) Grounded Cognition. Annual Review of Psychology, 59, 617-645. http://dx.doi.org/10.1146/annurev.psych.59.103006.093639

[49] Lake, B.M., Salakhutdinov, R. and Tenenbaum, J.B. (2012) Concept Learning as Motor Program Induction: A LargeScale Empirical Study. Proceedings of the 34th Annual Conference of the Cognitive Science Society, Sapporo, 1-4 August 2012, 659-664.

[50] Olshausen, B. and Field, D. (1996) Emergence of Simple-Cell Receptive Field Properties by Learning a Sparse Code for Natural Images. Nature, 381, 607-609. http://dx.doi.org/10.1038/381607a0

[51] Feldman, J. (2003) The Simplicity Principle in Human Concept Learning. Current Directions in Psychological Science, 12, 227-232. http://dx.doi.org/10.1046/j.0963-7214.2003.01267.x

[52] Gallant, S.I. and Okaywe, T.W. (2013) Representing Objects, Relations, and Sequences. Neural Computation, 25, 2038-2078. http://dx.doi.org/10.1162/NECO_a_00467

[53] Kanerva, P. (2009) Hyperdimensional Computing: An Introduction to Computing in Distributed Representation with High-Dimensional Random Vectors. Cognitive Computation, 1, 139-159.

[54] Gayler, R.W. (2003) Vector Symbolic Architectures Answer Jackendoff's Challenges for Cognitive Neuroscience. Proceedings of the ICCS/ASCS International Conference on Cognitive Science, Sydney, 13-17 July 2003, 133-138.

[55] Eliasmith, C., Stewart, T.C., Choo, X., Bekolay, T., DeWolf, T., Tang, Y. and Rasmussen, D. (2012) A Large-Scale Model of the Functioning Brain. Science, 338, 1202-1205. http://dx.doi.org/10.1126/science.1225266

[56] Kanerva, P. (1988) Sparse Distributed Memory. The MIT Press, Cambridge.

[57] Abbott, J.T., Hamrick, J.B. and Grifths, T.L. (2013) Approximating Bayesian Inference with a Sparse Distributed Memory System. Proceedings of the 35th Annual Conference of the Cognitive Science Society, Berlin, 31 July -3 August 2013, 6.

[58] Neumann, J. (2002) Learning the Systematic Transformation of Holographic Reduced Representations. Cognitive Systems Research, 3, 227-235. http://dx.doi.org/10.1016/S1389-0417(01)00059-6

[59] Curto, C. and Itskov, V. (2008) Cell Groups Reveal Structure of Stimulus Space. PLoS Computational Biology, 4, Article ID: e1000205.

[60] Kleyko, D., Lyamin, N., Osipov, E. and Riliskis, L. (2012) Dependable MAC Layer Architecture Based on Holographic Data Representation Using Hyper-Dimensional Binary Spatter Codes. Multiple Access Communications: 5th International Workshop, MACOM 2012, Springer, Berlin, 134-145.

[61] Jakimovski, P., Schmidtke, H.R., Sigg, S., Chaves, L.W.F. and Beigl, M. (2012) Collective Communication for Dense Sensing Environments. Journal of Ambient Intelligence and Smart Environments (JAISE), 4, 123-134.

[62] Hentrich, D., Oruklu, E. and Saniie, J. (2011) Polymorphic Computing: Definition, Trends, and a New Agent-Based Architecture. Circuits and Systems, 2, 358-364.

[63] Ramo, S. (1959) All about Polymorphics. https://archive.org/details/AllAboutPolymorphics1959

[64] Granacki, J.J. and Vahey, M.D. (2002) Monarch: A High Performance Embedded Processor Architecture with Two Native Computing Modes. Proceedings of High Performance Embedded Computing Workshop 2002, Lexington, 24-26 September 2002.

[65] Nasution, B.B. and Khan, A.I. (2008) A Hierarchical Graph Neuron Scheme for Real-Time Pattern Recognition. IEEE Transactions on Neural Networks, 19, 212-229. http://dx.doi.org/10.1109/TNN.2007.905857

[66] Osipov, E., Khan, A. and Amin, A.M. (2014) Holographic Graph Neuron. Proceedings of the 2nd International Conference on Computer and Information Sciences (ICCOINS 2014), Kuala Lumpur, 3-5 June 2014. 